\title{
GDF-15 como Biomarcador em Doenças Cardiovasculares
}

\section{GDF-15 as a Biomarker in Cardiovascular Disease}

\author{
Bruna Miers May, ${ }^{10}$ Mauricio Pimentel, ${ }^{2}$ Leandro Ioschpe Zimerman, ${ }^{2}{ }^{\circledR}$ Luis Eduardo Rohde ${ }^{2}$ \\ Universidade Federal do Rio Grande do Sul - Programa de Pós-Graduação em Ciências da Saúde: Cardiologia e Ciências Cardiovasculares, ${ }^{1}$ \\ Porto Alegre, RS - Brasil \\ Hospital de Clinicas de Porto Alegre, ${ }^{2}$ Porto Alegre, $R S$ - Brasil
}

\section{Resumo}

Nos últimos anos, vários biomarcadores estão ganhando importância clínica na avaliação diagnóstica e prognóstica de pacientes com doenças cardiovasculares. O fator de crescimento e diferenciação celular-15 (GDF-15) é uma citocina induzida por estresse e inflamação, membro da família do TGF-, cuja produção no miocárdio foi demonstrada experimentalmente em resposta à injúria isquêmica ou sobrecarga cardíaca. Este novo marcador foi positivamente correlacionado com aumento do risco de eventos cardiovasculares em estudos populacionais e configurou-se preditor independente de mortalidade e prognóstico adverso em pacientes com doença arterial coronariana e insuficiência cardíaca. Este trabalho tem como objetivo revisar o valor diagnóstico e prognóstico do GDF-15 em diferentes cenários na cardiologia.

\section{Introdução}

O fator de crescimento e diferenciação celular-15 (GDF15) é uma citocina da família do fator de transformação do crescimento beta (TGF-) encontrada em baixa quantidade nos tecidos e no plasma, exceto pela placenta e próstata. Antigamente chamado de citocina inibidora de macrófagos-1 (MIC-1), o GDF-15 foi descoberto há mais de 20 anos e assim nomeado devido a um possível papel antagonista após a ativação de macrófagos por citocinas inflamatórias (interleucinas e fator de necrose tumoral) em estudos experimentais. A sua função no organismo permanece incerta até hoje e pode variar conforme o tecido estudado. A expressão desse marcador é regulada por estresse e injúria tecidual, e está associada a condições inflamatórias em diferentes órgãos, inclusive no miocárdio. ${ }^{1}$

Em modelos animais, o GDF-15 apresentou-se inicialmente como uma proteína cardioprotetora, prevenindo morte celular,

\section{Palavras-chave}

Doenças Cardiovasculares; Biomarcadores; GDF-15 Fator de Diferenciação de Crescimento; Citocinas; Estresse; Inflamação; Prognóstico.

\footnotetext{
Correspondência: Mauricio Pimentel •

Hospital de Clinicas de Porto Alegre - Ramiro Barcelos, 2350.

CEP 90000-000, Porto Alegre, RS - Brasil

E-mail: mpimentelrs@gmail.com

Artigo recebido em 04/05/2020, revisado em 11/07/2020, aceito em 12/08/2020
}

DOI: https://doi.org/10.36660/abc.20200426 e dilatação e hipertrofia cardíaca. Expressão aumentada do marcador foi encontrada após estímulos agressores como sobrecarga de pressão e isquemia tecidual. ${ }^{2,3} \mathrm{~A}$ ativação da enzima NOS-2 (óxido nítrico sintase 2), em situações de estresse, participa na regulação positiva de GDF-15 por vias de sinalização intracelular dependentes de óxido nítrico. ${ }^{3}$ Em ratos geneticamente modificados para apresentarem deficiência de GDF-15, na isquemia miocárdica induzida, eram observadas áreas de infarto maiores com maior apoptose de miócitos, indicando uma possível função de limitação de dano miocárdico do marcador. ${ }^{3}$ A Figura 1 apresenta os principais fatores que influenciam a expressão do GDF-15.

Outro trabalho experimental correlacionou níveis elevados do GDF-15 em cardiomiócitos de ratos com uma redução na ativação do hormônio do crescimento $(\mathrm{GH})$, sugerindo sua participação na via de sinalização do $\mathrm{GH}$. Após essa descoberta, os mesmos autores realizaram estudo em crianças com cardiopatia congênita, encontrando níveis significativamente maiores de GDF-15 no plasma de crianças com cardiopatia e deficiência de crescimento, em relação a controles saudáveis e a cardiopatas com crescimento normal. ${ }^{4}$

Após os resultados dos trabalhos iniciais, o GDF-15 passou a ser investigado em vários contextos clínicos, apresentando-se na maior parte dos estudos como um biomarcador associado a maior risco de desenvolver eventos cardiovasculares. ${ }^{5-9}$ Atualmente, a dosagem sérica do GDF-15 encontra-se disponível comercialmente apenas na Europa, em outras regiões os kits são acessíveis apenas para fins de pesquisa clínica e experimental. ${ }^{10} \mathrm{~A}$ dosagem é realizada por imunoensaios, ensaios por quantificação de complexos antígeno-anticorpo marcados por radioisótopos (técnica de imunorradiometria - IRMA), por enzimas (ELISA) ou luminescentes (quimioluminescência). A faixa de detecção varia entre 400-20000 ng/L, com boa precisão e reprodutibilidade (taxas de imprecisão intra e inter-ensaios abaixo de 10\%). O método mais utilizado hoje é o por ELISA, pelo menor custo e maior acessibilidade. ${ }^{11,12}$

O objetivo deste artigo é revisar o papel do GDF-15 em diferentes cenários da cardiologia, avaliando-se a possibilidade de sua incorporação como biomarcardor no diagnóstico e estratificação de risco de cardiopatias prevalentes.

\section{Risco Cardiovascular em Indivíduos Saudáveis}

O primeiro estudo em humanos a relacionar o GDF-15 com doença cardiovascular foi publicado em 2002 e incluiu 27 628 mulheres saudáveis acompanhadas por quatro anos. Os resultados demonstraram um aumento de 2,7 vezes no risco de evoluir com eventos cardiovasculares (infarto, acidente 


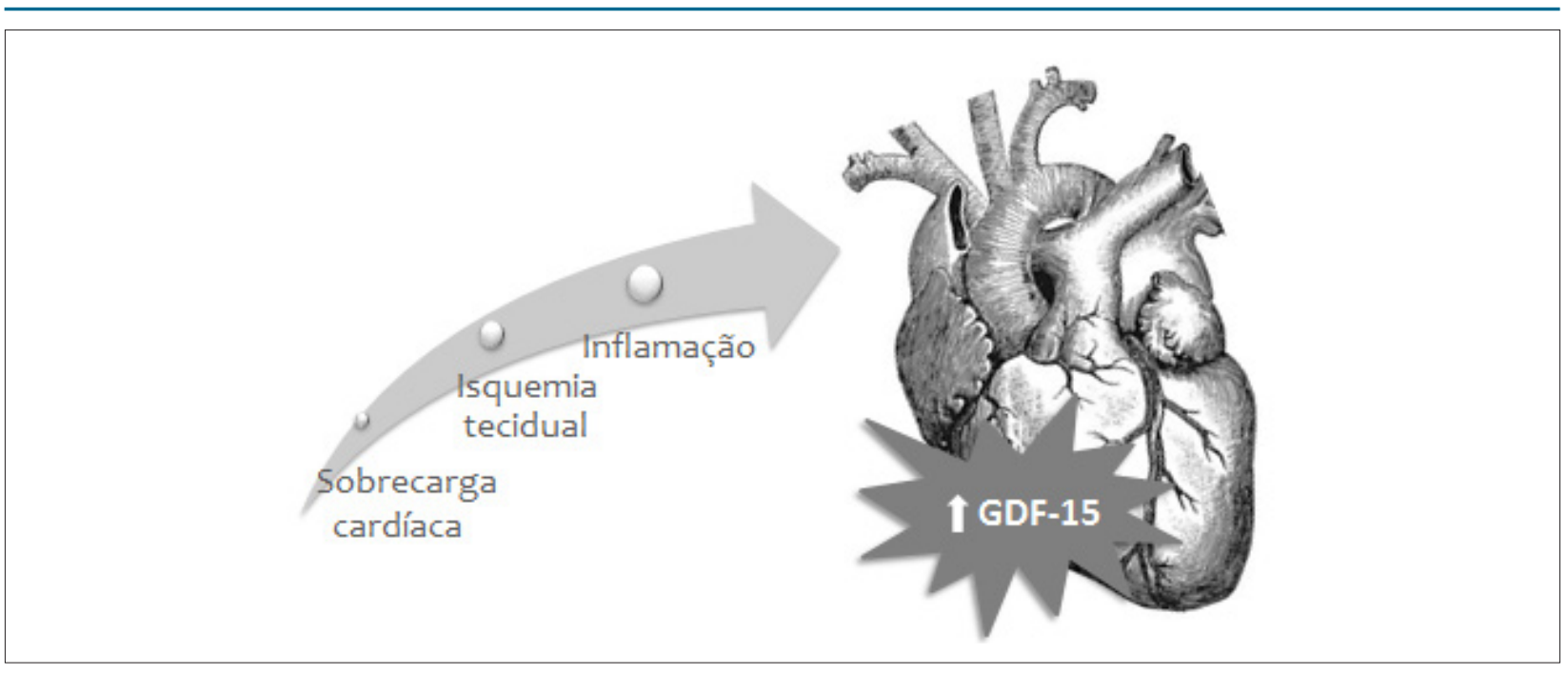

Figura 1 - Fatores que modificam a expressão do fator de crescimento e diferenciação celular-15(GDF-15) no sistema cardiovascular.

vascular cerebral e morte cardiovascular) nas participantes com níveis da citocina acima de $856 \mathrm{ng} / \mathrm{L} .{ }^{13} \mathrm{Em}$ uma coorte com 1391 pacientes sem doença cardiovascular estabelecida, o marcador foi preditor independente de mortalidade por todas as causas e de morte cardiovascular com hazard ratio (HR) de 1,5 (IC 95\%: 1,3-1,8), com poder discriminatório comparável ao peptídeo natriurético cerebral (BNP) (HR 1,3; IC 95\%: 1,2-1,5). ${ }^{14}$

Dados do Framingham Heart Study, onde foram avaliados 85 biomarcadores (incluindo BNP, PCR e GDF-15) em 3523 participantes ao longo de 14 anos de seguimento, mostraram que o GDF-15 foi o único marcador, em análise multivariada, a manter associação significativa com os três desfechos avaliados: eventos cardíacos ateroscleróticos (HR 1,43; IC 95\%: 1,20-1,58), insuficiência cardíaca (IC) (HR 2,08; IC 95\%: 1,72-2,53) e mortalidade (HR 1,96; IC 95\%: 1,76-2,17). ${ }^{8}$

\section{Doença Arterial Coronariana (DAC)}

O GDF-15 foi estudado em pacientes admitidos no hospital após síndrome coronariana aguda (SCA) e em portadores de doença coronariana estável.

\section{Síndromes Coronarianas Agudas}

Pacientes que apresentavam níveis aumentados de GDF-15 em dosagem realizada na internação devido à SCA evoluíram com um número maior de eventos como morte cardiovascular, reinfarto e acidente vascular cerebral em 12 meses de seguimento após a alta, demostrando um valor prognóstico com relação à progressão de doença aterosclerótica. ${ }^{15}$

Outro estudo observacional recente demonstrou a mesma associação prognóstica do marcador com eventos cardiovasculares maiores (mortalidade total, infarto não fatal e internação por IC). Porém, em análise multivariada ajustada para outros fatores de risco, o GDF15 permaneceu significativo apenas para mortalidade e desenvolvimento de IC. ${ }^{16}$
Ainda no contexto de doença aguda, um ensaio clínico avaliando estratégia invasiva versus conservadora na SCA sem supradesnivelamento do segmento ST encontrou incidência significativa mais alta de eventos nos pacientes com níveis elevados de GDF-15 alocados no grupo da estratégia conservadora. Os autores sugerem que a dosagem do marcador possa complementar os escores de risco na seleção daqueles que se beneficiam mais da estratégia invasiva precoce. ${ }^{17}$

Corroborando com essa ideia, o uso do escore GRACE, ajustado para GDF-15, associado à medida dos níveis de GDF15 na admissão ao hospital, aumentou a acurácia do escore [área sob a curva (AUC) ROC de 0,79 para 0,85]. Entre os pacientes que não apresentaram eventos durante o seguimento de 6 meses, 54 foram classificados como risco intermediário pelo escore de GRACE e teriam sido reclassificados em baixo risco se utilizado o escore ajustado. ${ }^{18}$ Tzikas et al., ${ }^{19}$ encontraram que o GDF-15 é preditor independente de eventos cardiovasculares de modo semelhante à troponina, com forte correlação com a gravidade da doença coronariana avaliada pelo escore Syntax após cateterismo cardíaco. ${ }^{19}$

Avaliando-se especificamente pacientes com infarto agudo do miocárdio com supradesnivelamento do segmento ST (IAMCSST) tratados com angioplastia primária, a taxa de mortalidade em 10 anos após o evento agudo aumentou de $6 \%$ para $19 \%$ nos pacientes com GDF-15 acima da mediana. ${ }^{20}$ Outro estudo avaliou a alteração dinâmica do GDF-15 durante as primeiras 24 horas de um evento de IAMCSST e mostrou pico da citocina em 12 horas, e manutenção dos níveis elevados até final das 24 horas. Níveis maiores na dosagem realizada após 24 horas se correlacionaram à maior mortalidade em 30 dias. ${ }^{21}$ Em relação à extensão do infarto e prognóstico, quanto maior a dosagem do marcador, maior o risco de remodelamento e dilatação ventricular em 12 meses. ${ }^{22}$ Estudo prospectivo analisou 92 biomarcadores em 847 pacientes com doença coronariana acompanhados por seis anos após infarto agudo. GDF-15 foi um dos dois únicos marcadores com poder para predizer mortalidade, após ajuste para fatores clínicos. ${ }^{23}$ 
Em metanálise incluindo oito estudos com pacientes acompanhados após uma SCA, o GDF-15 foi considerado um forte preditor de mortalidade com risco relativo (RR) de 6,08 (IC 95\%: 4,79-7,71; p < 0,001) e reinfarto não-fatal, com RR de 1,76 (IC 95\%: 1,49-2,07; p < 0,001). ${ }^{24}$ Uma metanálise mais recente com 13 estudos e um total de 43547 pacientes com SCA corrobora esses resultados: RR para mortalidade de 6,75 (IC 95\%: 5,81-7,84; $p<0,001$ ) e para reinfarto não-fatal 1,95 (IC 95\%: 1,72-2,21, p < 0,001)..$^{25}$

Ainda no cenário de SCAs, em que está indicado o uso de dupla antiagregação plaquetária, o GDF-15 também foi preditor de risco de sangramento. ${ }^{15} \mathrm{Em}$ uma análise posthoc do ensaio clínico PLATO (ticagrelor x clopidogrel no IAMCSST), foi identificado um risco três vezes mais alto de sangramento nos pacientes que mantém níveis do marcador acima de 1800 ng/L em dosagem realizada um mês após a SCA, independentemente da droga utilizada. ${ }^{26}$ Nesse contexto, em que os níveis de GDF-15 mantêm-se elevados após o evento agudo, um marcador de risco de sangramento pode ajudar na decisão de manter a terapia antitrombótica dupla além do tempo usualmente recomendado.

\section{Doença Coronariana Estável}

Na doença coronariana crônica, GDF-15 foi dosado em uma coorte com 14577 pacientes portadores de angina estável, revascularização prévia, e doença multiarterial ou infarto há mais de um ano. Ao longo do seguimento, níveis acima de 1827 ng/L associaram-se a maior risco de morte cardiovascular (HR 2,63; IC 95\%: 1,9-3,6; $p<0,001$ ), morte súbita cardíaca (HR 3,06; IC 95\%: 1,9-4,8; $p<0,001)$ e hospitalização por IC (HR 5,8; IC 95\%: 3,2-10; p = 0,006), de forma independente a outros marcadores como troponina, proteína $\mathrm{C}$ reativa e BNP. Neste estudo, ${ }^{27}$ o GDF-15 não se correlacionou com novo evento trombótico após ajuste para os demais biomarcadores.

\section{Insuficiência Cardíaca (IC)}

O GDF-15 foi avaliado em diversas coortes de pacientes portadores de IC e comparado, na maioria, a peptídeos natriuréticos [BNP ou fragmento $\mathrm{N}$-terminal do pró-hormônio do BNP (NT-proBNP)]. A principal diferença entre eles é a proporção do aumento no plasma conforme o tipo de disfunção ventricular. O NT-proBNP, um marcador de estresse hemodinâmico do ventrículo esquerdo, está aumentado na IC com fração de ejeção reduzida de forma mais significativa do que na IC com fração de ejeção preservada. Em contrapartida, o GDF-15 encontra-se elevado de forma semelhante na disfunção sistólica e diastólica, sugerindo que injúria inflamatória seja parte da fisiopatologia de ambas as condições. O GDF-15 apresentou-se como importante preditor de eventos adversos e mortalidade, independentemente da fração de ejeção e nível sérico de NT-proBNP.28-33

\section{IC com Fração de Ejeção Teduzida (ICFEr)}

A avaliação do GDF-15 em diferentes estágios da IC define-o como um biomarcador de evolução de doença, aumentando exponencialmente conforme a piora de classe funcional e o remodelamento do ventrículo esquerdo. Os níveis de GDF-15 já estão elevados na fase pré-clínica da IC (estágio B) e a associação do marcador ao NT-proBNP aumentou a acurácia diagnóstica para IC, inclusive nessa fase inicial. ${ }^{34} \mathrm{Na}$ mesma direção, outro estudo prospectivo correlacionou o GDF-15 à progressão da disfunção ventricular e perda de capacidade funcional em pacientes com fração de ejeção menor que 35\%, encontrando níveis séricos progressivamente maiores conforme a gravidade da IC. Este resultado se manteve significativo após ajuste para outros fatores de risco como o consumo de oxigênio de pico (VO2 pico), idade e taxa de filtração glomerular. ${ }^{35}$

O primeiro grande estudo que avaliou o valor prognóstico do GDF-15 na ICFEr foi realizado utilizando-se a base de dados do ensaio clínico Val-HeFT (Valsartan Heart Failure Trial), que avaliou o uso de valsartan em pacientes com IC. O GDF-15 foi avaliado no início do estudo $(n=1734)$ e após 12 meses de seguimento $(n=1517)$. No início do estudo, 85\% dos pacientes apresentavam valores de GDF-15 elevados (>1200ng/ml). Em análise multivariada incluindo variáveis clínicas, $\mathrm{BNP}$, troponina e proteína $\mathrm{C}$ reativa, valores elevados de GDF-15 permaneceram associados de forma independente com aumento do risco de mortalidade total (HR 1,007; IC 95\%: 1,001-1,014; p=0,02), mas não com a ocorrência de primeiro evento mórbido (HR 1,003; IC 95\%: 0,997-1,008; $p=0,34)$, que incluía morte, morte súbita com ressuscitação, hospitalizações por IC, ou necessidade de uso de inotrópicos ou vasodilatadores intravenosos por mais de $4 \mathrm{~h}$ sem hospitalização. Em 12 meses de seguimento, os valores de GDF-15 aumentaram de modo semelhante no grupo placebo e no grupo valsartan, sendo associados de modo independente com mortalidade total e primeiro evento mórbido. Este achado sugere que o GDF-15 represente um eixo fisiopatológico que não é afetado pelas terapias prescritas.

Mais recentemente, o GDF-15 foi estudado em 1935 pacientes incluídos no estudo PARADIGM-HF, que comparou sacubitril/valsartana versus enalapril em pacientes com ICFEr. O GDF-15 basal e os seus níveis em um e oito meses de tratamento foram associados significativamente com aumento do risco de mortalidade total e de eventos cardiovasculares. Cada incremento de 20\% no GDF-15 basal foi associado com maior risco de mortalidade total (HR 1,13; IC 95\%:1,08-1,18; $\mathrm{p}<0,001)$, desfecho combinado de morte cardiovascular ou hospitalização por IC (HR 1,09; IC 95\%: 1,05-1,14; P<0,001) e morte por IC (HR 1,16; IC 95\% 1,05-1,28; p<0,001). O incremento no nível sérico de GDF-15 durante o estudo não foi influenciado pelos tratamentos prescritos. ${ }^{36}$

O papel do marcador foi estudado ainda em pacientes submetidos a implante de ressincronizador cardíaco. Durante o seguimento de 158 pacientes, 72\% apresentaram boa resposta à ressincronização, mas aqueles em que o nível sérico basal do GDF-15 era superior a $2720 \mathrm{ng} / \mathrm{L}$ tiveram risco significativamente maior de morte cardiovascular e reinternações por IC em 2,5 anos. Apesar de se demonstrar o valor prognóstico do biomarcador nesta população, o nível basal e a variação em um ano pós-implante não foram capazes de predizer resposta ao dispositivo. ${ }^{37}$

No cenário de doença avançada, foram dosados cinco biomarcadores (PCR, NT-proBNP, GDF-15, galectina-3 e troponina) em pacientes com classe funcional NYHA (New 
York Heart Association) III. Entre eles, o GDF-15 foi o melhor preditor de mortalidade a longo prazo, inclusive com maior valor preditivo que NT-proBNP (AUC 0,78 versus 0,63). ${ }^{38}$

Em pacientes com miocardiopatia não-isquêmica grave, GDF-15 foi analisado em biópsias realizadas durante implante de dispositivos de assistência ventricular ou transplante cardíaco, e se mostrou fortemente correlacionado ao grau de fibrose miocárdica nessas amostras. ${ }^{39}$ Nesta coorte, observouse que um mês após o implante do suporte circulatório, os níveis do marcador reduziram-se significativamente comparado ao pré-implante, sugerindo mais uma vez a sua associação com o grau de disfunção miocárdica. ${ }^{39}$

\section{IC com Fração de Ejeção Preservada (ICFEp)}

Atualmente, os critérios diagnósticos para ICFEp são baseados principalmente em sintomas de IC e alterações ecocardiográficas sugerindo elevação nas pressões de enchimento cardíacas. Ainda assim, há bastante heterogeneidade nos conceitos e critérios adotados pelas Sociedades e no diagnóstico de consultório na prática clínica. Em pacientes com ICFEp, foram detectados níveis elevados de GDF-15, e sua associação direta com a relação E/e' no ecocardiograma. A combinação de NT-proBNP e GDF15 elevados aumentou a acurácia diagnóstica (atingindo uma AUC de 0,93) para ICFEp. ${ }^{40}$ Ainda, estudos de coorte prospectivos com esta população demonstraram que quanto maior o nível sérico do GDF-15, maior o grau de disfunção diastólica e pior a classe funcional NYHA. ${ }^{41,42}$

Um desafio diagnóstico é a definição de ICFEp em obesos mórbidos, devido a limitações ecocardiográficas como janela desfavorável, dispneia multifatorial e níveis reduzidos de BNP. No estudo de Baessler et al., ${ }^{43}$ em pacientes com índice de massa corporal acima de $30 \mathrm{~kg} / \mathrm{m}^{2}$, o GDF-15 se correlacionou com elevação nas pressões de enchimento ao ecocardiograma. A adição do GDF-15 aos critérios ecocardiográficos de disfunção diastólica obteve melhor performance diagnóstica nessa população, comparada à associação do BNP aos mesmos critérios (AUC 0,76 x AUC 0,56 , respectivamente). ${ }^{43}$

\section{IC Agudamente Descompensada}

A concentração sérica do GDF-15 na admissão de pacientes agudamente descompensados é elevada (a maioria dos estudos encontrou valores acima de $1200 \mathrm{ng} / \mathrm{L}$ ). Quanto mais alta a concentração do marcador ou se os níveis aumentam durante a internação, maior o risco de reinternações por IC e mortalidade após a alta hospitalar. ${ }^{44,45}$

Estudo $^{46}$ com 55 pacientes portadores de ICFEr realizou dosagens seriadas de diversos biomarcadores durante a internação por descompensação cardíaca e 30 dias após a alta, demonstrando que a curva do GDF-15 se assemelha à de dois outros marcadores: ST2 (biomarcardor pertencente à família dos receptores de interleucina-1) e BNP. Neste estudo, um rápido decréscimo no nível do marcador é evidenciado com a melhora clínica dos pacientes, diferente do que ocorreu com outras proteínas inflamatórias como proteína $\mathrm{C}$ reativa, TNF-alfa, IL-6, galectinas e mieloperoxidase. ${ }^{46}$
Modelos construídos adicionando o GDF-15 a marcadores clássicos como troponina e BNP demonstram que a sua dosagem na IC aguda acrescenta valor prognóstico. Esse dado sugere a presença de várias vias fisiopatológicas independentes em pacientes hospitalizados por IC e novamente denota relevância clínica do marcador nesse cenário. ${ }^{47,48}$

A Figura 2 apresenta as principais correlações do GDF-15 com diferentes aspectos clínicos da IC.

\section{Morte Súbita}

O GDF-15 também foi estudado no contexto de estratificação de risco de morte súbita em pacientes com doenças cardiovasculares. No cenário de doença coronariana estável, demonstrou-se que pacientes com GDF-15 elevado apresentam maior risco de morte súbita com razão de risco (hazard ratio) de 3,0 (IC 95\%: 1,94-4,84; p < 0,001). ${ }^{27}$

Em um estudo coorte recente, realizou-se a dosagem de ST2 e GDF-15 em 52 pacientes portadores de IC de etiologia não isquêmica, acompanhados por um tempo médio de sete anos. O GDF-15 correlacionou-se com um aumento em duas vezes no risco de morte por arritmia e morte súbita com ressuscitação (HR 2,2; IC 95\% 1,1-4,5; p=0,028) e foi superior ao ST2 em predizer mortalidade por qualquer causa (HR 2,4; IC 95\%: 1,4-4,2; $p=0,003$ versus HR 1,6; IC 95\%: $1,05-2,7 ; p=0,03) \cdot{ }^{49}$

\section{Fibrilação Atrial (FA)}

Em pacientes com FA, em tratamento adequado e anticoagulados, aqueles com níveis mais elevados do marcador apresentaram taxas 4 a 5 vezes mais altas de mortalidade, independentemente da idade, sexo e escore $\mathrm{CHA}_{2} \mathrm{DS}_{2} \mathrm{VASC}^{50}{ }^{50}$ Resultado semelhante foi encontrado por Sharma et al., ${ }^{51}$ sendo o GDF-15 fortemente associado à morte por progressão da IC e sangramento. ${ }^{51}$

Pacientes portadores de FA não valvar, não anticoagulados, cujos níveis de GDF-15 sérico são acima de 809 ng/dL têm mais risco de ter trombo no átrio esquerdo, independente de idade, volume do átrio e $\mathrm{CHA}_{2} \mathrm{DS}_{2}$ VASc. ${ }^{52}$

Em um estudo com 14.798 pacientes anticoagulados, foi encontrado um risco 3,5 vezes mais elevado de sangramento maior nos participantes com GDF-15 elevado, independente da terapia antitrombótica utilizada e demais comorbidades. ${ }^{50}$ Após esse achado, foi desenvolvido nesta população e validado em outra amostra semelhante o escore de risco ABC (baseado em idade, biomarcadores - GDF-15, hemoglobina e troponina - e história clínica de sangramento), para sangramentos, sendo o GDF-15 o integrante de maior contribuição para o risco. Esse escore obteve melhor acurácia que o escore mais utilizado na prática clínica (HAS-BLED). ${ }^{53}$

\section{Doença Renal Crônica}

Remodelamento cardíaco, fibrose e inflamação são possíveis fatores envolvidos no aumento da incidência de eventos cardiovasculares em pacientes com doença renal crônica (DRC).

Avaliando-se biomarcadores possivelmente representativos dessas condições, demonstrou-se que as proteínas ST2, 


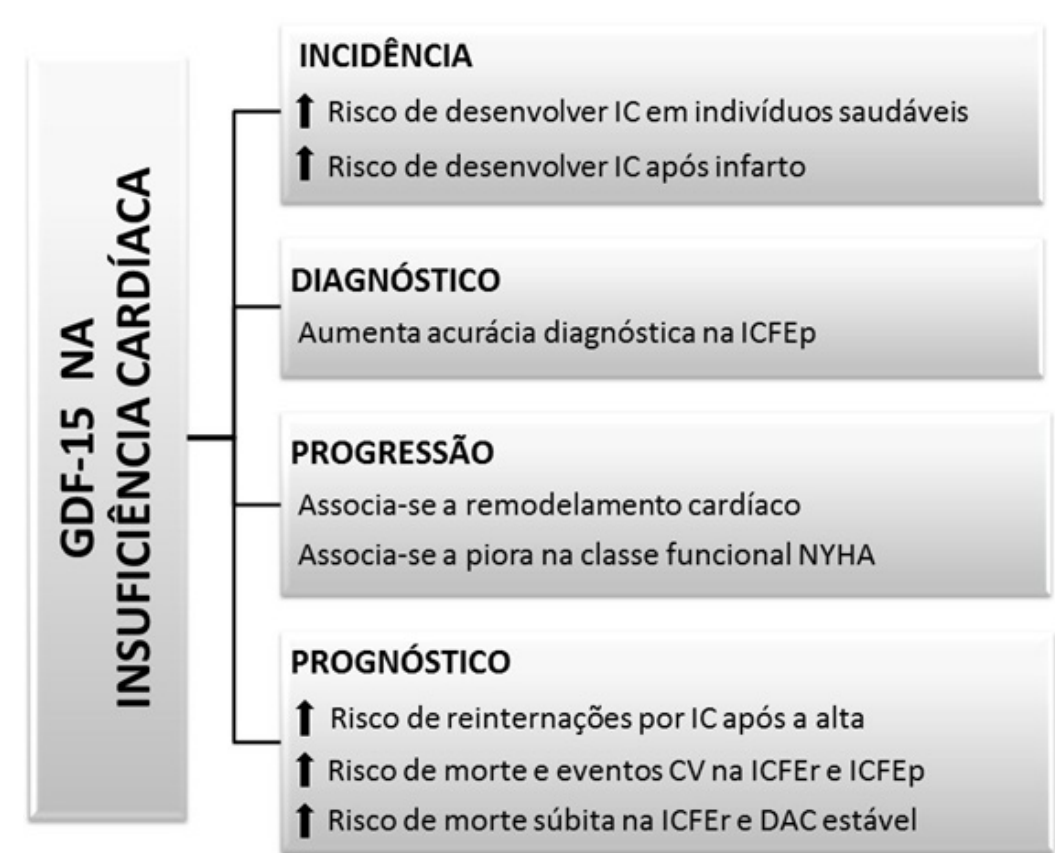

Figura 2 - Implicação do aumento nos níveis de fator de crescimento e diferenciação celular-15 (GDF-15) em diferentes aspectos clínicos na insuficiência cardíaca. IC: insuficiência cardiaca; CV: cardiovasculares; ICFEp: insuficiência cardiaca com fração de ejeção preservada; NYHA: New York Heart Association; ICFEr: insuficiência cardiaca com fração de ejeção reduzida; DAC: doença arterial coronariana

galectina-3 e GDF-15 associaram-se significativamente à mortalidade nestes pacientes, mas não a eventos ateroscleróticos. Entre esses, apenas o GDF-15 correlacionouse ao risco de desenvolver IC..$^{54}$

Resultado semelhante foi encontrado por Bansal et al., ${ }^{55} \mathrm{em}$ que o GDF-15 foi preditor de IC em pacientes com disfunção renal, assim como o NT-proBNP. Entretanto, ao contrário do peptídeo natriurético, o GDF-15 teve uma correlação mais forte com ICFEp. ${ }^{55}$

Pacientes com taxa de filtração glomerular abaixo de $60 \mathrm{~mL} / \mathrm{min} / 1,73 \mathrm{~m}^{2}$ apresentam níveis significativamente maiores de GDF-15 e NT-proBNP, quando comparados a pacientes com função renal normal. Em uma coorte com 358 pacientes com DRC e disfunção sistólica, o GDF-15 refinou a estratificação prognóstica de pacientes com NT-proBNP baixo e foi fortemente associado a eventos adversos, de maneira mais significativa que o próprio peptídeo. ${ }^{56}$

Na Tabela 1 encontram-se os valores, em média, dos níveis séricos de GDF-15 associados às condições clínicas exploradas nesta revisão.

\section{Conclusão}

O GDF-15 é um biomarcador sérico cuja expressão parece ser afetada por estresse, injúria tecidual e inflamação, embora seu eixo fisiopatológico ainda não seja completamente entendido. Estudos observacionais com pessoas saudáveis demonstraram associação do GDF-15 com maior risco de eventos cardiovasculares ao longo do tempo. Em pacientes com doença arterial coronariana e IC, o GDF-15 correlacionou-se a um risco elevado de mortalidade total e eventos adversos. A incorporação do GDF-15 melhorou a performance diagnóstica da ICFEp e contribuiu para a elaboração de escore mais acurado para risco de sangramento na FA. A utilização do GDF-15 na prática clínica como marcador prognóstico e sua capacidade de orientar a tomada de decisão clínica depende ainda de novos estudos com maior número de pacientes.

\section{Contribuição dos Autores}

Concepção e desenho da pesquisa, Obtenção de dados e Análise e interpretação dos dados: May BM, Pimentel M; Redação do manuscrito e Revisão crítica do manuscrito quanto ao conteúdo intelectual importante: May BM, Pimentel M, Zimerman LI, Rohde LE.

\section{Potencial Conflito de Interesses}

Declaro não haver conflito de interesses pertinentes.

\section{Fontes de Financiamento}

O presente estudo não teve fontes de financiamento externas.

\section{Vinculação Acadêmica}

Este artigo é parte de dissertação de Mestrado de Bruna Miers May pela Universidade Federal do Rio Grande do Sul - Programa de Pós-Graduação em Ciências da Saúde: Cardiologia e Ciências Cardiovasculares. 


\begin{tabular}{|c|c|c|c|c|c|}
\hline \multirow{2}{*}{$\begin{array}{l}\text { Valor para } \\
\text { diagnóstico }\end{array}$} & & \multicolumn{4}{|c|}{ Predição de eventos adversos } \\
\hline & & $\begin{array}{c}\text { Eventos } \\
\text { cardiovasculares }\end{array}$ & $\begin{array}{c}\text { Morte } \\
\text { cardiovascular }\end{array}$ & Morte súbita & Mortalidade total \\
\hline IC com fração de ejeção reduzida ${ }^{7,33,34}$ & $>1200$ & $>2040$ & $>2252$ & * & $>2040$ \\
\hline IC com fração de ejeção preservada ${ }^{31,40,42}$ & $>1160$ & * & NA & NA & * \\
\hline Síndrome coronariana aguda ${ }^{15,19}$ & $>967$ & $>1550$ & $>1550$ & NA & $>1259$ \\
\hline Doença arterial coronariana estável ${ }^{27}$ & NA & $>1253$ & $>1827$ & $>1253$ & $>915$ \\
\hline
\end{tabular}

NA: Não avaliável (sem estudos para aquele desfecho ou estudos pequenos não confiáveis). * Uso do biomarcador como variável contínua, sem ponto de corte especifico.

\section{Referências}

1. Emmerson PJ, Duffin KL, Chintharlapalli S, Wu X. GDF15 and Growth Control. Front Physiol. 2018;9:1-7.

2. Xu J, Kimball TR, Lorenz JN, Brown DA, Bauskin AR, Klevitsky R, et al. GDF15/MIC-1 functions as a protective and antihypertrophic factor released from the myocardium in association with SMAD protein activation. Circ Res. 2006;98(3):342-50.

3. Kempf T, Eden M, Strelau J, Naguib M, Willenbockel C, Tongers J, et al. The transforming growth factor-superfamily member growth-differentiation factor-15 protects the heart from ischemia/reperfusion injury. Circ Res. 2006;98(3):351-60.

4. Wang T, Liu J, McDonald C, Lupino K, Zhai X, Wilkins BJ, et al. GDF 15 is a heart-derived hormone that regulates body growth. EMBO Mol Med. 2017;9(8):1150-64.

5. Wollert KC, Kempf T, Wallentin L. Growth differentiation factor 15 as a biomarker in cardiovascular disease. Clin Chem. 2017;63:140-51.

6. Khan SQ, Ng K, Dhillon O, Kelly D, Quinn P, Squire IB, et al. Growth differentiation factor-15 as a prognostic marker in patients with acute myocardial infarction. Eur Heart J. 2009;30(9):1057-65.

7. Anand IS, Kempf T, Rector TS, Tapken H, Allhoff T, Jantzen F, et al. Serial measurement of growth-differentiation factor-15 in heart failure: Relation to disease severity and prognosis in the valsartan heart failure trial. Circulation. 2010;122(14):1387-95.

8. HoJE, Lyass A, Courchesne P, Chen G, Liu C, Yin X, et al. Protein biomarkers of cardiovascular disease and mortality in the community. J Am Heart Assoc. 2018;7(14):e008108.

9. Xie S, Lu L, Liu L. Growth differentiation factor-15 and the risk of cardiovascular diseases and all-cause mortality: A meta-analysis of prospective studies. Clin Cardiol. 2019;42(5):513-23.

10. Chaikijurajai T, Tang WHW. Reappraisal of Inflammatory Biomarkers in Heart Failure. Curr Heart Fail Rep. 2020 Feb;17(1):9-19.

11. Wollert KC, Kempf T, Giannitsis E, Bertsch T, Braun SL, Maier H, et al. An Automated Assay for Growth Differentiation Factor 15. J Appl Lab Med An AACC Publ. 2017;1(5):510-21.

12. Kempf T, Horn-Wichmann R, Brabant G, Peter T, Allhoff T, Klein G, et al. Circulating concentrations of growth-differentiation factor 15 in apparently healthy elderly individuals and patients with chronic heart failure as assessed by a new immunoradiometric sandwich assay. Clin Chem. 2007;53(2):284-91.

13. Brown DA, Breit SN, Buring J, Fairlie WD, Bauskin AR, Liu T, et al. Concentration in plasma of macrophage inhibitory cytokine-1 and risk of cardiovascular events in women: a nested case-control study. Lancet. 2002 Jun;359(9324):2159-63.

14. Daniels LB, Clopton P, Laughlin GA, Maisel AS, Barrett-Connor E. Growthdifferentiation factor-15 is a robust, independent predictor of 11-year mortality risk in community-dwelling older adults: The rancho bernardo study. Circulation. 2011;123(19):2101-10.

15. Hagström E, James SK, Bertilsson M, Becker RC, Himmelmann A, Husted $S$, et al. Growth differentiation factor-15 level predicts major bleeding and cardiovascular events in patients with acute coronary syndromes: Results from the PLATO study. Eur Heart J. 2016;37(16):1325-33.

16. Peiró ÓM, García-Osuna Á, Ordóñez-Llanos J, Cediel G, Bonet G, Rojas $\mathrm{S}$, et al. Long-term prognostic value of growth differentiation factor-15 in acute coronary syndromes. Clin Biochem. 2019 Nov;73:62-69.

17. Wollert KC, Kempf T, Lagerqvist B, Lindahl B, Olofsson S, Allhoff T, et al. Growth differentiation factor 15 for risk stratification and selection of an invasive treatment strategy in non-ST-elevation acute coronary syndrome. Circulation. 2007;116(14):1540-8.

18. Widera C, Pencina MJ, Meisner A, Kempf T, Bethmann K, Marquardt I, et al. Adjustment of the GRACE score by growth differentiation factor 15 enables a more accurate appreciation of risk in non-ST-elevation acute coronary syndrome. Eur Heart J. 2012;33(9):1095-104.

19. Tzikas S, Palapies L, Bakogiannis C, Zeller T, Sinning C, Baldus S, et al. GDF-15 predicts cardiovascular events in acute chest pain patients. PLoS One. 2017;12(8):1-13.

20. Bodde MC, Hermans MPJ, van der Laarse A, Mertens B, Romijn FPHTM, Schalij MJ, et al. Growth Differentiation Factor-15 Levels at Admission Provide Incremental Prognostic Information on All-Cause Long-term Mortality in ST-Segment Elevation Myocardial Infarction Patients Treated with Primary Percutaneous Coronary Intervention. Cardiol Ther. 2019;8(1):29-41.

21. Rueda F, Lupón J, García-garcía C, Cediel G, Nevado MCA, Gregori JS, et al. Acute-phase dynamics and prognostic value of growth differentiation factor-15 in ST-elevation myocardial infarction. Clin Chem Lab Med. 2019 Jun;57(7):1093-1101.

22. Dominguez-Rodriguez A, Abreu-Gonzalez P, Avanzas P. Relation of growthdifferentiation factor 15 to left ventricular remodeling in ST-segment elevation myocardial infarction. Am J Cardiol. 2011 Oct; 108(7):955-8.

23. Skau E, Henriksen E, Wagner P, Hedberg P, Siegbahn A, Leppert J. GDF-15 and TRAIL-R2 are powerful predictors of long-term mortality in patients with acute myocardial infarction. Eur J Prev Cardiol. 2017;24(15):1576-83.

24. Zhang S, Dai D, Wang X, Zhu H, Jin H, Zhao R, et al. Growth differentiation factor-15 predicts the prognoses of patients with acute coronary syndrome: A meta-analysis. BMC Cardiovasc Disord. 2016;16(1):1-7.

25. Wang Y, Zhen C, Wang R, Wang G. Growth-differentiation factor-15 predicts adverse cardiac events in patients with acute coronary syndrome: A meta-analysis. Am J Emerg Med. 2019;37(7):1346-52. 
26. Lindholm D, Hagström E, James SK, Becker RC, Cannon CP, Himmelmann A, et al. Growth Differentiation Factor 15 at 1 Month After an Acute Coronary Syndrome Is Associated With Increased Risk of Major Bleeding. J Am Heart Assoc. 2017;6(4):e005580.

27. Hagström E, Held C, Stewart RAH, Aylward PE, Budaj A, Cannon CP, et al. Growth differentiation factor 15 predicts all-cause morbidity and mortality in stable coronary heart disease. Clin Chem. 2017;63(1):325-33.

28. Van Kimmenade RRJ, Januzzi JL. Emerging biomarkers in heart failure. Clin Chem. 2012;58(1):127-38

29. Sinning C, Zengin E, Zeller T, Schnabel RB, Blankenberg S, Westermann D. Candidate biomarkers in heart failure with reduced and preserved ejection fraction. Biomarkers. 2015;20(4):258-65.

30. Sinning C, Kempf T, Schwarzl M, Lanfermann S, Ojeda F, Schnabel RB, et al. Biomarkers for characterization of heart failure - Distinction of heart failure with preserved and reduced ejection fraction. Int J Cardiol. 2017;227:272-7.

31. Chan MMY, Santhanakrishnan R, Chong JPC, Chen Z, Tai BC, Liew OW, et al. Growth differentiation factor 15 in heart failure with preserved vs. reduced ejection fraction. Eur J Heart Fail. 2016;18(1):81-8.

32. Santhanakrishnan R, Chong JPC, Ng TP, Ling LH, Sim D, Toh G. Leong K, et al. Growth differentiation factor 15, ST2, high-sensitivity troponin T, and $\mathrm{N}$-terminal pro brain natriuretic peptide in heart failure with preserved vs. reduced ejection fraction. Eur J Heart Fail. 2012;14(12):1338-47.

33. Sharma A, Stevens SR, Lucas J, Fiuzat M, Adams KF, Whellan DJ, et al. Utility of Growth Differentiation Factor-15, A Marker of Oxidative Stress and Inflammation, in Chronic Heart Failure: Insights From the HF-ACTION Study. JACC Hear Fail. 2017;5(10):724-34.

34. Li J, Cui Y, Huang A, Li Q, Jia W, Liu K, et al. Additional Diagnostic Value of Growth Differentiation Factor-15 (GDF-15) to N-Terminal B-Type Natriuretic Peptide (NT-proBNP) in Patients with Different Stages of Heart Failure. Med Sci Monit. 2018 Jul;24:4992-9.

35. Rullman E, Melin M, Mandić M, Gonon A, Fernandez-Gonzalo R, Gustafsson T. Circulatory factors associated with function and prognosis in patients with severe heart failure. Clin Res Cardiol. 2020;109(6):655-672.

36. Bouabdallaoui N, Claggett B, Zile MR, McMurray JJV, O'Meara E, Packer $M$, et al. Growth differentiation factor-15 is not modified by sacubitril/ valsartan and is an independent marker of risk in patients with heart failure and reduced ejection fraction: the PARADIGM-HF trial. Eur J Heart Fail. 2018;20(12):1701-9.

37. Foley PWX, Stegemann B, Ng K, Ramachandran S, Proudler A, Frenneaux MP, et al. Growth differentiation factor-15 predicts mortality and morbidity after cardiac resynchronization therapy. Eur HeartJ. 2009;30(22):2749-57.

38. Lok DJ, Klip IT, Lok SI, Porte PWB De, Badings E, Van Wijngaarden J, et al. Incremental Prognostic Power of Novel Biomarkers Protein, Galectin-3, and High-Sensitivity Troponin-T) in Patients With Advanced Chronic Heart Failure. Am J Cardiol. 2013;112(6):831-7.

39. Lok SI, Winkens B, Goldschmeding R, Van Geffen AJP, Nous FMA, Van Kuik J, et al. Circulating growth differentiation factor- 15 correlates with myocardial fibrosis in patients with non-ischaemic dilated cardiomyopathy and decreases rapidly after left ventricular assist device support. Eur J Heart Fail. 2012;14(11):1249-56.

40. Stahrenberg R, Edelmann F, Mende M, Kockskämper A, Düngen HD, Lüers $\mathrm{C}$, et al. The novel biomarker growth differentiation factor 15 in heart failure with normal ejection fraction. Eur J Heart Fail. 2010;12(12):1309-16.

41. Dinh W, Füth R, Lankisch M, Hess G, Zdunek D, Scheffold T, et al. Growthdifferentiation factor-15: A novel biomarker in patients with diastolic dysfunction? Arq Bras Cardiol. 2011;97(1):65-75
42. Izumiya Y, Hanatani S, Kimura Y. Growth Differentiation Factor-15 Is a Useful Prognostic Marker in Patients With Heart Failure With Preserved Ejection Fraction. Can J Cardiol. 2014;30(3):338-44.

43. Baessler A, Strack C, Rousseva E, Wagner F, Bruxmeier J, Schmiedel M, et al. Growth-differentiation factor-15 improves reclassification for the diagnosis of heart failure with normal ejection fraction in morbid obesity. Eur J Heart Fail. 2012;14(11):1240-8.

44. Cotter G, Voors AA, Prescott MF, Felker GM, Filippatos G, Greenberg BH, et al. Growth differentiation factor 15 (GDF-15) in patients admitted for acute heart failure: Results from the RELAX-AHF study. Eur J Heart Fail. 2015;17(11):1133-43.

45. Jankovic-Tomasevic R, Pavlovic SU, Jevtovic-Stoimenov T, Apostolovic S, Stanojevic D, Jovanovic I, et al. Prognostic utility of biomarker growth differentiation factor- 15 in patients with acute decompensated heart failure. Acta Cardiol. 2016;71(5):587-95.

46. Boulogne M, Sadoune M, Launay JM, Baudet M, Cohen-Solal A, Logeart D. Inflammation versus mechanical stretch biomarkers over time in acutely decompensated heart failure with reduced ejection fraction. Int I Cardiol. 2017;226:53-9.

47. Demissei BG, Cotter G, Prescott MF, Felker GM, Filippatos G, Greenberg $\mathrm{BH}$, et al. A multimarker multi-time point-based risk stratification strategy in acute heart failure: results from the RELAX-AHF trial. Eur J Heart Fail. 2017;19(8):1001-10

48. Bettencourt P, Ferreira-Coimbra J, Rodrigues P, Marques P, Moreira H, Pinto $\mathrm{MJ}$, et al. Towards a multi-marker prognostic strategy in acute heart failure: a role for GDF-15. ESC Hear Fail. 2018;5(6):1017-22.

49. Stojkovic S, Kaider A, Koller L, Brekalo M, Wojta J, Diedrich A, et al. GDF15 is a better complimentary marker for risk stratification of arrhythmic death in non-ischaemic, dilated cardiomyopathy than soluble ST2. J Cell Mol Med. 2018;22(4):2422-9.

50. Wallentin L, Hijazi Z, Andersson U, Alexander JH, De Caterina R Hanna M, et al. Growth differentiation factor 15, a marker of oxidative stress and inflammation, for risk assessment in patients with atrial fibrillation: Insights from the Apixaban for reduction in stroke and other thromboembolic events in atrial fibrillation (ARISTOTLE). Circulation. 2014;130(21):1847-58.

51. Sharma A, Hijazi Z, Andersson U, Al-Khatib SM, Lopes RD, Alexander $\mathrm{JH}$, et al. Use of biomarkers to predict specific causes of death in patients with Atrial fibrillation: Insights from the Aristotle Trial. Circulation. 2018;138(16):1666-76.

52. Hu XF, Zhan R, Xu S, Wang J, Wu J, Liu X, et al. Growth differentiation factor 15 is associated with left atrial/left atrial appendage thrombus in patients with nonvalvular atrial fibrillation. Clin Cardiol. 2018;41(1):34-8.

53. Hijazi Z, Oldgren J, Lindbäck J, Alexander JH, Connolly SJ, Eikelboom JW, et al. The novel biomarker-based ABC (age, biomarkers, clinical history)bleeding risk score for patients with atrial fibrillation: a derivation and validation study. Lancet. 2016;387(10035):2302-11.

54. Tuegel C, Katz R, Alam M, Bhat Z, Bellovich K, de Boer I, et al. GDF-15, Galectin 3, Soluble ST2, and Risk of Mortality and Cardiovascular Events in CKD. Am J Kidney Dis. 2018 Oct; 72(4):519-28.

55. Bansal N, Zelnick L, Go A, Anderson A, Christenson R, Deo R, et al. Cardiac Biomarkers and Risk of Incident Heart Failure in Chronic Kidney Disease: The CRIC (Chronic Renal Insufficiency Cohort) Study. J Am Heart Assoc. 2019;8(21):1448-57.

56. Benes J, Kotrc M, Wohlfahrt P, Conrad MJ, Franekova J, Jabor A, et al. The Role of GDF-15 in Heart Failure Patients With Chronic Kidney Disease. Can J Cardiol. 2019;35(4):462-70. 